\title{
Temperature dependence of the threshold and Auger recombination in asymmetric quantum-well heterolasers
}

\author{
Igor A. Sukhoivanov ${ }^{\mathrm{a}, \mathrm{b}}$, Olga V. Mashoshyna ${ }^{*}$, Valerii K. Kononenko ${ }^{\mathrm{c}}$, Dmitrii V. Ushakov ${ }^{\mathrm{c}}$ \\ ${ }^{a}$ Guanajuato Univ., Salamanca, Mexico \\ ${ }^{\mathrm{b}}$ Kharkov National Univ. of Radio Electronics, 61166, Lenin Ave., 14, Kharkov, Ukraine \\ ${ }^{\mathrm{c}}$ Stepanov Inst. of Physics NASB, Fr. Scorina Pr., 70, 220072 Minsk, Belarus
}

\begin{abstract}
The temperature dependence of the lasing threshold in the GaInAs-GaInAsP-InP bi-quantum-well heterolasers with different widths of the quantum wells $(4$ and $9 \mathrm{~nm}$ ) has been determined. Processes of non-radiative Auger recombination which occur in the active region of the quantum-well lasers have been included into consideration. The analytical approach for the evaluation of the characteristic temperature of the lasing threshold is presented. For described asymmetric quantumwell heterostructure lasers, it is shown that the influence of Auger recombination processes on the temperature behavior of the lasing threshold is not essential until the temperature of the active region is lower than $360 \mathrm{~K}$ and the cavity losses do not exceed $80 \mathrm{~cm}^{-1}$.
\end{abstract}

Keywords: asymmetric quantum-well laser, Auger recombination, threshold, gain, temperature parameters.

\section{INTRODUCTION}

Basic components of modern telecommunication systems are lasers operating at the wavelength $1.55 \mu \mathrm{m}$ where the optical fiber losses are minimal. The laser diodes must be stable and have low values of the threshold current. At the operation temperature enhancement, the lasing threshold increases mainly because of the Auger recombination (AR) processes accelerating [1]. While the long-wavelength lasers development it is important to get the weakening of such processes. A plausible way for solving this problem can be application of the semiconductor lasers based on asymmetric quantum-well heterostructures [2]. These laser heterostructures can serve as broadband light sources for multi-channel fiber-optical network systems [3].

In the work, the temperature dependence of the lasing threshold in the GaInAs-GaInAsP-InP bi-quantum-well heterolasers with different widths of the quantum wells $(4$ and $9 \mathrm{~nm}$ ) has been determined. Including into consideration the processes of non-radiative $A R$ allows to take into account the non-equilibrium processes which occur in the active region of the quantum-well lasers more completely and accurately.

\section{BACKGROUND THEORY}

\subsection{Lasing threshold}

In the general case, the threshold current in quantum-well heterostructure lasers $j_{\text {th }}$ is determined by the threshold quantity of the spontaneous radiative recombination rate $R_{\mathrm{sp}}$ in the active region, i. e.,

$$
j_{\mathrm{th}}=\frac{e d}{\eta^{\prime} \eta_{\mathrm{sp}}} R_{\mathrm{sp}},
$$

where $d$ is the quantum well width, $\eta$ ' is the injection efficiency, $\eta_{\mathrm{sp}}$ is the quantum yield of spontaneous emission. The coefficient $\eta$ ' describes the escape of injected current carriers to emitters and depends on the laser structure design, doping,

"mashoshina@kture.kharkov.ua; phone 38005770 21484; fax 3800577021013 
and temperature. The quantity of $\eta_{\mathrm{sp}}$ characterizes the relative role of useless recombination processes in the active region, such as transitions through different defects and non-radiative AR. Increasing the temperature influences on the threshold value of the current carrier concentration that has, accordingly, an effect on $\eta$ ' and $\eta_{\mathrm{sp}}$ and on $R_{\mathrm{sp}}$ as well. In asymmetric quantum-well heterostructures, the additional complex behavior appears due to electron-optical interaction of the quantum wells in the conditions of non-uniform excitation of the active region [2]. In this case, tunneling and carrier transport through modified barriers between the quantum wells provide specific changes in the threshold versus temperature. Below, conditions of uniform excitation of the quantum wells with varied width are only examined.

Quantum-mechanical analysis of non-equilibrium electron processes in quantum wells of the lasers shows that at direct dipole optical transitions the threshold $j_{\text {th }}$ changes practically directly proportional the operation temperature $T$ where loses in the cavity are not very high [4]. It can be used as a first step for the evaluation of the lower value of the lasing threshold. Then, roughly we have $j_{\mathrm{th}} \approx a T / \eta^{\prime} \eta_{\mathrm{sp}}$, where $a=2 k e^{3} \mu n_{0} E_{g}^{2} / 3 \pi c^{3} \hbar^{4}, \mu$ is the numerical factor, $n_{0}$ is the refractive index, $E_{g}$ is the energy gap. The factor $\mu$ in general is about of 0.7 and the coefficient $a$ for different laser materials covers the interval of 0.2 to $3 \mathrm{~A} / \mathrm{cm}^{2} \mathrm{~K}$.

The violation of conservation of the electron wave vector at optical transitions provides a second power dependence of $j_{\mathrm{th}} \sim T^{2}[4,5]$. Involving highlying excited subbands at the transitions increases power index of the dependence $j_{\mathrm{th}}(T)$ and therefore functionally $j_{\mathrm{th}} \sim T^{n}$, where $n$ lies in the range of 1 to 3 [4]. The temperature decreasing of $\eta^{\prime}$ and $\eta_{\mathrm{sp}}$ has obviously a definite effect too. Additional behavior of the lasing threshold is introduced certainly by temperature changes in the cavity losses.

Often, the temperature dependence of the threshold is described by an exponential function with the characteristic parameter $T_{0}$. However, though it is a convenient empirical parameter, its value is ordinary indefinite, depends on the temperature operation interval, and widely varies for the same laser diode type. According to the determining of $T_{0}$ one has at a narrow temperature operation interval $T \approx T_{\text {op }}$ approximately $T_{0} \approx T_{\text {op }} / n$.

To exclude the AR rate, we present the threshold in the form

$$
j_{\mathrm{th}}=\frac{e}{\eta^{\prime}}\left(A n_{\mathrm{th}}^{n}+C n_{\mathrm{th}}^{3}\right)
$$

where $n_{\text {th }}$ is the threshold sheet concentration of electrons. The coefficient $A$ is determined by the optical transition probability and for direct dipole transitions $(n=1)$ it can be presented as the Einstein coefficient $A_{c v}$, i. e., in this case, $A \approx A_{c v} \approx 1 / \tau_{\mathrm{sp}}$, where $\tau_{\mathrm{sp}}$ is the threshold lifetime of current carriers at spontaneous radiative recombination [4]. In the limit case of no k-selection rule $(n=2)$, the coefficient $A$ is also related to $A_{c v}$ by a definite relation [5].

The last term in Eq. (2) describes the AR rate. As a rule, the AR coefficient $C$ is determined in the activation approach, i. e., $C \approx C_{0} \exp \left(-E_{\text {act }} / k T\right)[1]$. The activation energy is related to the energy of transitions between corresponding subband levels $E_{q}$ through the formula $E_{\text {act }} \approx E_{q} \Delta m$, where $\Delta m$ is the effective mass relation that is different for each of the AR processes. Generally, procedure of the determining of $C$ in quantum-well heterostructures, especially for strained laser systems, is rather complex and difficult [6].

If $n_{\mathrm{th}} \sim T$, then we obtain

$$
T_{0} \approx \frac{T_{\mathrm{op}}}{n+\left(1-\eta_{\mathrm{sp}}\right)\left(3-n+E_{\mathrm{act}} / k T_{\mathrm{op}}\right)} .
$$

As seen, if the role of the AR becomes essential $\left(\eta_{\mathrm{sp}}<<1\right)$, the relation between the activation energy $E_{\text {act }}$ and the thermal energy $k T_{\text {op }}$ determines the value of $T_{0}$. On the other hand, from experimental results on the measurements of $T_{0}$ it is possible to evaluate the effective value of the activation energy of the AR processes. 


\subsection{Energy band structure}

Numerical calculations have been performed for the asymmetric bi-quantum-well heterostructure lasers in the GaInAsGaInAsP-InP system. The active region includes two quantum wells of widths 4 and $9 \mathrm{~nm}$ (Fig. 1). Energy levels in the quantum wells have been preliminarily determined in the effective mass approximation using standard parameters for semiconductor components [7] (Tabl. 1). In the quantum well of the 4-nm width, only one subband of electrons and light holes and two subbands of heavy holes are realized, in the quantum well of the 9-nm width the number of possible electron and light hole subbands is one more and the number of heavy hole subbands becomes up to four. The laser diodes operate at the wavelength near $1.55 \mu \mathrm{m}$ and provide a widen tunable gain spectrum.

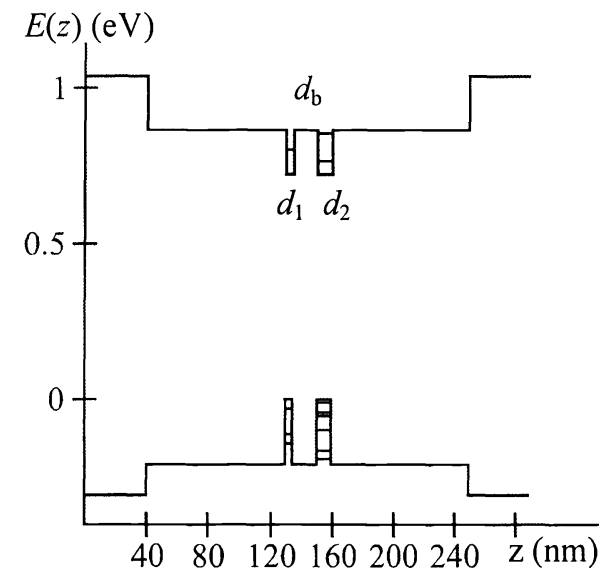

(a)

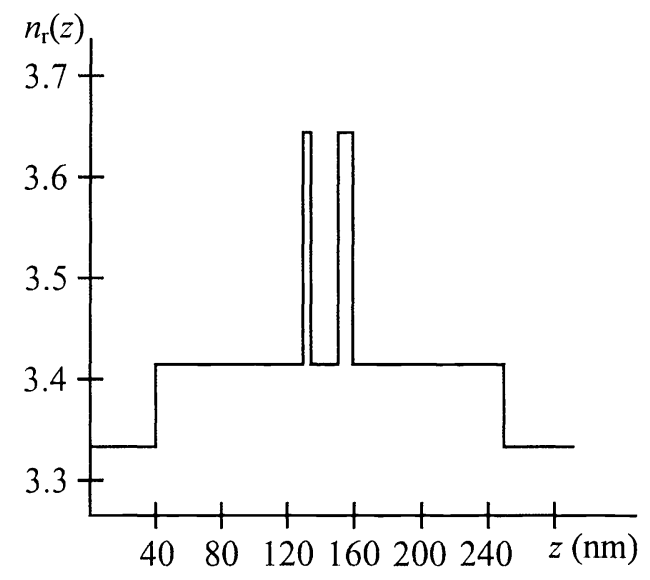

(b)

Figure 1: Configuration of (a) the energy bands $E(z)$ and (b) profile of the refractive index $n_{r}(z)$ in the active region of the $\mathrm{Ga}_{0.47} \mathrm{In}_{0.53} \mathrm{As}-\mathrm{Ga}_{0.18} \mathrm{In}_{0.82} \mathrm{As}_{0.4} \mathrm{P}_{0.6}-\mathrm{InP}$ heterostructure laser. Subband levels in different quantum wells of widths $d=4$ and $9 \mathrm{~nm}$ are also schematically shown.

Table 1: Parameters for the calculations in the effective mass approximation, $T=300 \mathrm{~K}$.

\begin{tabular}{|c|c|c|c|c|c|c|c|c|c|c|}
\hline \multicolumn{2}{|c|}{ Energy gap $E_{g}, \mathrm{eV}$} & \multicolumn{2}{|c|}{ Width } & \multicolumn{2}{|c|}{ Band offset, $\mathrm{eV}$} & \multicolumn{5}{|c|}{ Effective mass in units $m_{e}$} \\
\hline \multirow{2}{*}{$\begin{array}{c}\text { quantum } \\
\text { well }\end{array}$} & \multirow[t]{2}{*}{ barrier } & \multirow{2}{*}{$\begin{array}{c}\text { quantum } \\
\text { wells }\end{array}$} & \multirow{2}{*}{$\begin{array}{l}\text { barrier } \\
d_{\mathrm{b}}, \mathrm{nm}\end{array}$} & \multirow{2}{*}{$\Delta E_{c}$} & \multirow{2}{*}{$\Delta E_{v}$} & electrons & \multicolumn{2}{|c|}{ heavy holes } & \multicolumn{2}{|c|}{ light holes } \\
\hline & & & & & & $m_{c}$ & $m_{v h}$ & $m_{\mathrm{vht}}$ & $m_{v l}$ & $m_{v l t}$ \\
\hline 0.718 & 1.060 & $\begin{array}{l}d_{1}=4 \mathrm{~nm}, \\
d_{2}=9 \mathrm{~nm}\end{array}$ & 15 & 0.137 & 0.205 & 0.043 & 0.307 & 0.051 & 0.040 & 0.116 \\
\hline
\end{tabular}

More detailed energy band structure in the quantum wells was calculated according to the four-band $\mathbf{k} \cdot \mathbf{p}$ method [8,9]. Dispersion energy curves for the hole subbands are shown in Fig. 2. These data were used for determining the gain and spontaneous radiative recombination spectra.

\subsection{Gain and radiative emission spectra}

The gain coefficient at the light frequency $v$ and a definite TE- or TM-polarization in the quantum well of the width $d_{i}$ $(i=1,2)$ is given as follows $[9,10]$

$$
\begin{gathered}
k(v)=\frac{e^{2}}{\varepsilon_{0} c m_{e}^{2} n_{0} v d_{i}} \sum_{n, m} \int L\left(h v-E_{c v}\right) d E_{c v} \times \\
\times \int\left|\mathbf{M}_{n m}\right|^{2}\left[f_{e}\left(E_{c n}\left(k_{\square}\right)\right)+f_{h}\left(E_{v m}\left(k_{\square}\right)\right)-1\right] \frac{d k_{\square}}{(2 \pi)^{2}} \delta\left(E_{c v}-E_{c n}\left(k_{\square}\right)+E_{v m}\left(k_{\square}\right)\right)
\end{gathered}
$$


where $n_{0}$ is the refractive index of the active region, $f_{e}$ and $f_{h}$ are Fermi-Dirac functions for electrons and holes, $E_{c n}\left(k_{5}\right)$ and $E_{v m}(k)$ are energies of the states involved in the optical transitions, $\left|\mathbf{M}_{n m}\right|^{2}$ is the squared matrix element of the dipole transitions between electron and hole subbands with the quantum numbers $n$ and $m$ accordingly. Here, the Lorentzian spectral broadening of the line emission is assumed and it is described by the function $L\left(h v-E_{c v}\right)$, where $E_{c v}$ is the energy of direct optical transitions between electron and hole subbands. The spectral broadening parameter $\Gamma_{c v}$ is considered, as ordinary, to be of $10 \mathrm{meV}$ [10]. Mention, that the squared matrix element of the transitions $\left|\mathbf{M}_{n m}\right|^{2}$ depends on the light polarization and reflects character of the involved hole states and effects of their mixing.

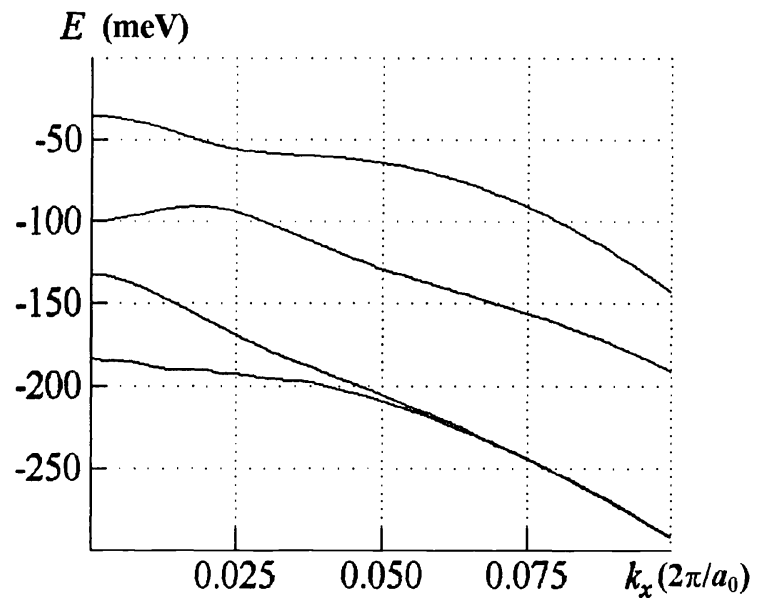

(a)

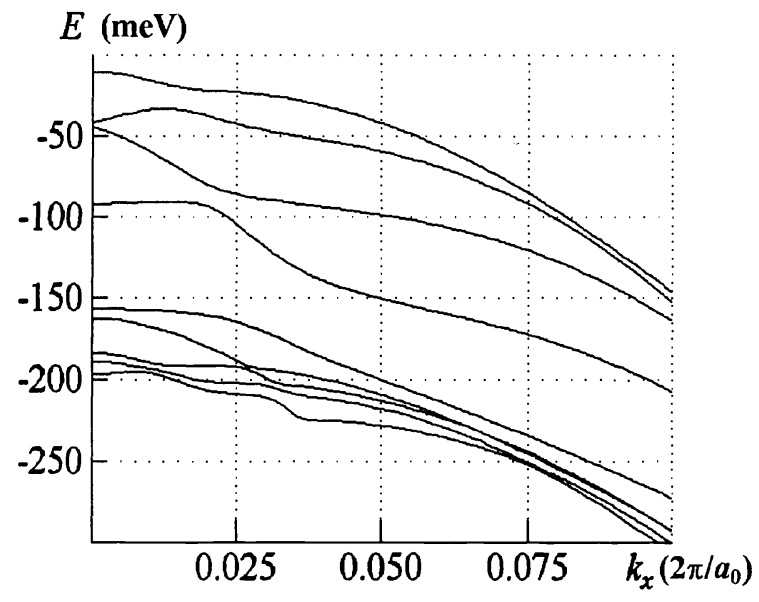

(b)

Figure 2: Distribution of energy states $E\left(k_{x}\right)$ in the valence band for quantum wells of the width (a) $d_{1}=4 \mathrm{~nm}$ and (b) $d_{2}=9 \mathrm{~nm} . T=300 \mathrm{~K}$. The wave vector component $k_{x}$ is given in units $2 \pi / a_{0}, a_{0}$ is the GaInAs crystal lattice period, $k_{y}=0$.

The spontaneous radiative recombination spectrum $r_{\mathrm{sp}}(h v)$ is calculated similar to $k(v)$ with the exception of that the matrix element of the transitions is taken as for the isotropic radiation, i. e., $\left|\mathbf{M}_{n m}\right|^{2}$ in Eq. (4) is replaced by the value of the squared matrix element of the interband transitions $\left|\mathbf{M}_{c v}\right|^{2}=m_{c}{ }^{2} E_{g} / 6 m_{c}$. Therefore, the spectral distribution of the spontaneous radiative recombination rate $r_{\mathrm{sp}}(h v)$ in the quantum well of the width $d_{i}$ is given in the form

$$
\begin{gathered}
r_{s p}(h v)=\frac{8 \pi e^{2} n_{0} v}{\varepsilon_{0} h m_{e}^{2} c^{3} d_{i}} \sum_{n, m} \int L\left(h v-E_{c v}\right) d E_{c v} \times \\
\times \int\left|\mathbf{M}_{c v}\right|^{2} f_{e}\left(E_{c n}\left(k_{\square}\right)\right) f_{h}\left(E_{v m}\left(k_{\square}\right)\right) \frac{d k_{\square}}{(2 \pi)^{2}} \delta\left(E_{c v}-E_{c n}\left(k_{\square}\right)+E_{v m}\left(k_{\square}\right)\right)
\end{gathered}
$$

The total spontaneous recombination rate $R_{\mathrm{sp}}$ is obtained by the summation of $r_{\mathrm{sp}}(h v)$ over all possible energies of the emitted quanta $h v$.

The calculated spectra of the gain and spontaneous emission are shown in Fig. 3. In this case, the difference of the quasiFermi levels which determines the excitation of the heterolaser active region equals $\Delta F=0.96 \mathrm{eV}$ and corresponds to the conditions, where the total gain $g\left(\lambda_{\mathrm{st}}\right)$ at the lasing wavelength $\lambda_{\mathrm{st}}=1.47 \mu \mathrm{m}$ reaches the value of the cavity losses $k_{1}$ up to $60 \mathrm{~cm}^{-1}$. As seen, both the wider quantum well gives a twice larger contribution in the total spontaneous recombination rate. As concerning the gain in the spectral region near the lasing wavelength, the contribution of the narrower quantum well is a half as much again the contribution of the wider quantum well. The waveguide gain at the threshold in a definite quantum well $(i=1,2)$ is determined by both the gain coefficient $k\left(\lambda_{\mathrm{st}}\right)$ and the optical confinement factor $\Gamma_{i}$. The value of the optical confinement factor can be evaluated in the equivalent three-layer waveguide model. Using standard parameters for the described asymmetric quantum-well heterostructure (Tabl. 1), we have for the TE-mode $\Gamma_{1}=3.5 \times 10^{-3}$ and $\Gamma_{2}=7.9 \times 10^{-3}$. Thought the optical localization of the electromagnetic wave is better in the 9-nm width quantum well, the value of $k\left(\lambda_{\mathrm{st}}\right)$ is more higher in the 4-nm width quantum well. 


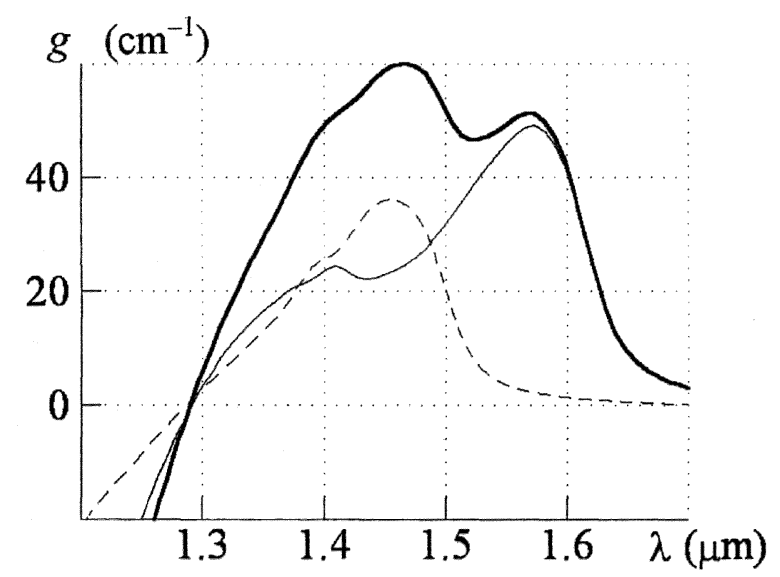

(a)

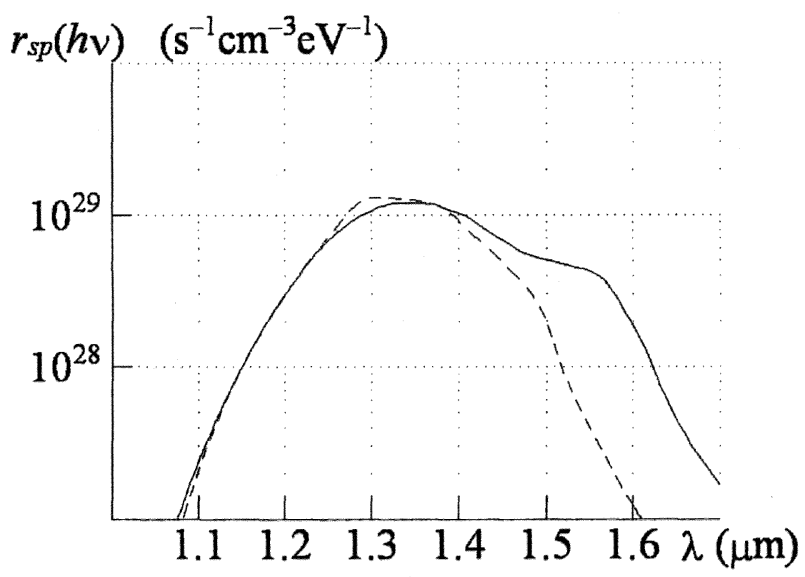

(b)

Figure 3: Spectra of (a) the gain $g(\lambda)$ at the TE-mode and (b) spontaneous radiative recombination $r_{\mathrm{sp}}(h v)$ at the excitation level $\Delta F=0.96 \mathrm{eV} . \mathrm{T}=300 \mathrm{~K}, k_{l}=60 \mathrm{~cm}^{-1}$. Dashed curves correspond with $d_{1}=4 \mathrm{~nm}$, fine curves correspond with $d_{2}=9 \mathrm{~nm} . \Gamma_{1}=3.5 \times 10^{-3}, \Gamma_{2}=7.9 \times 10^{-3}$, sheet concentrations of electrons in the quantum wells are $n_{1}=1.23 \times 10^{13} \mathrm{~cm}^{-2}$ and $n_{2}=2.06 \times 10^{13} \mathrm{~cm}^{-2}$, spontaneous recombination rates are $R_{\mathrm{spl}}=0.75 \times 10^{22} \mathrm{~s}^{-1} \mathrm{~cm}^{-2}$ and $R_{\mathrm{sp} 2}=1.89 \times 10^{22} \mathrm{~s}^{-1} \mathrm{~cm}^{-2}$.

\subsection{Temperature dependencies}

Numerical calculations show that with increasing the temperature of the laser diode active region the concentration of nonequilibrium current carriers in the quantum wells at the threshold increases approximately linearly with $T$ (Fig. 4, a). The coefficient of the linearity depends on the width $d$ and losses $k_{l}$. The threshold rate of the spontaneous radiative recombination in the quantum wells increases linearly with increasing the temperature as well. Accordingly, the lasing threshold current density $j_{\mathrm{th}}$ follows a similar law.

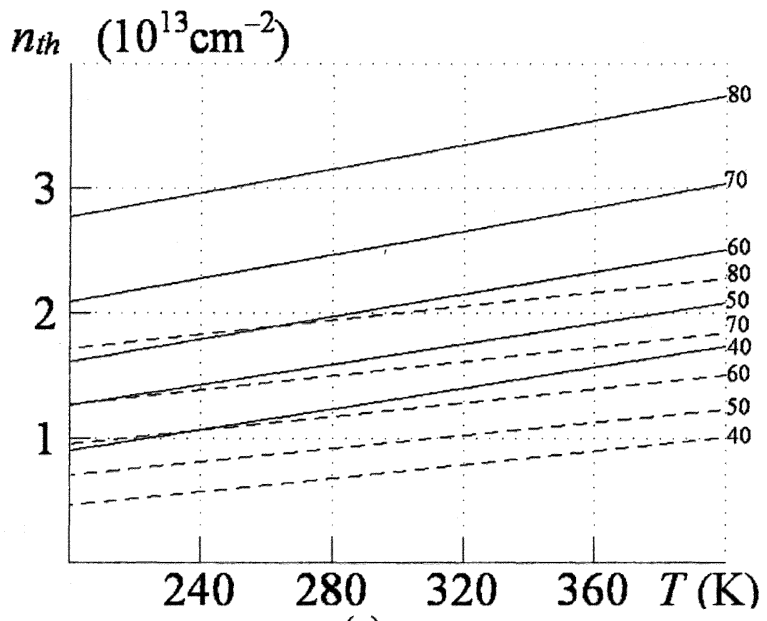

(a)

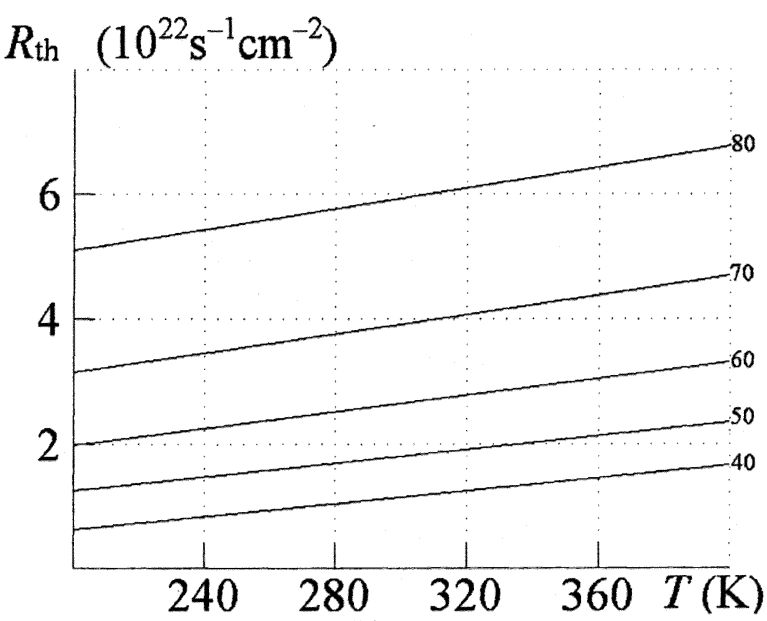

(b)

Figure 4: Temperature dependencies of (a) the threshold concentrations $n_{\mathrm{th}}$ in the quantum wells and (b) total radiative recombination rate $R_{\text {th }}$ at different losses $k_{1}$ (values on the curves in $\mathrm{cm}^{-1}$ ). Dashed curves correspond with $d_{1}=4 \mathrm{~nm}$, solid curves correspond with $d_{2}=9 \mathrm{~nm} . \Gamma_{1}=3.5 \times 10^{-3}, \Gamma_{2}=7.9 \times 10^{-3}$.

Mention, that the dependence $j_{t h}(T)$ is markedly determined by the losses because of the power function $j_{t h}\left(k_{1}\right)$. At the operation temperature $T=300 \mathrm{~K}$ and low losses $k_{1}=40 \mathrm{~cm}^{-1}$, the lasing threshold is about of $1.8 \mathrm{kA} / \mathrm{cm}^{2}$. As seen from Fig. $4, \mathrm{~b}$, the threshold $j_{\text {th }}=e R_{\text {th }}$ becomes rather high at larger losses $k_{l}$, e. g., at losses $k_{1}=70 \mathrm{~cm}^{-1}$, the value $j_{\text {th }}$ is equal to $6.2 \mathrm{kA} / \mathrm{cm}^{2}$. At the higher operation temperatures and larger losses the lasing threshold grows up to $10.9 \mathrm{kA} / \mathrm{cm}^{2}$. Therewith, the threshold concentration of non-equilibrium current carriers in the quantum wells markedly increases. As a result, contribution of the AR rate in the lasing threshold has to enhance the temperature dependence $j_{\text {th }}(T)$. 


\subsection{Auger recombination rate}

Calculations of the AR rate in the laser active region have been made according to the conventional method [1]. The thickness of the barrier layer of $15 \mathrm{~nm}$ allows to ignore the tunneling processes that simplifies estimations. The next interactions were considered: three electrons and one heavy hole $(\mathrm{CCCH})$, one electron, two heavy holes and one light hole (CHHL), one electron, two heavy holes and one split-off-band hole (CHHS), and one electron, two light holes and one splitoff-band hole (CLLS). Initially, evaluations of the AR rate $R$ are carried out for each quantum well separately (Figs. 5 and 6), then the obtained results are included in the determining of the lasing threshold versus the temperature. Values of the current carrier concentration $n / d$ were varied from $1 \times 10^{19}$ to $7.5 \times 10^{19} \mathrm{~cm}^{-3}$ (depending on the quantum well width) in the temperature range from 250 to $350 \mathrm{~K}$, assuming the conditions of the electroneutrality in the laser active region.

The activation energy $E_{\text {act }}$ is related to the energy of transitions between corresponding subband levels $E_{q}$ through the formula $E_{\text {act }}=E_{q} \Delta m$ and can be presented in the following expressions for each processes CCCH, CHHL, CHHS, and CLLS, respectively,

$$
\begin{gathered}
E_{\mathrm{act}}=E_{h} \frac{m_{c}}{m_{c}+m_{v h t}}, \quad E_{\mathrm{act}}=E_{l} \frac{m_{v l t}}{m_{c}+2 m_{v h t}-m_{v l t}}, \\
E_{\mathrm{act}}=\left(E_{h}-\Delta\right) \frac{m_{v s}}{m_{c}+2 m_{v h t}-m_{v s}}, \quad E_{\mathrm{act}}=\left(E_{l}-\Delta\right) \frac{m_{v s}}{m_{c}+2 m_{v l t}-m_{v s}},
\end{gathered}
$$

where $m_{c}$ is the effective mass of electrons, $m_{v i t}$ is the transverse component of the effective mass of heavy or light holes ( $i=h$ or $l), m_{v s}$ is the effective mass of holes in the split-off valence band, $E_{h}$ and $E_{l}$ are the transition energies between electron and heavy and light hole states, $\Delta$ is the split-off separation energy. Data of calculated parameters are presented in Tabl. 2.

Table 2: Activation parameters for different AR processes in the quantum wells, $T=300 \mathrm{~K}, m_{v s}=0.118 m_{\mathrm{e}}, \Delta=0.361 \mathrm{eV}$.

\begin{tabular}{|c|c|c|c|c|c|}
\hline \multirow{2}{*}{$\begin{array}{c}\text { AR process } \\
\text { type }\end{array}$} & $\begin{array}{c}\text { Effective mass } \\
\text { relation } \Delta m\end{array}$ & \multicolumn{2}{|c|}{$d_{1}=4 \mathrm{~nm}$} & \multicolumn{2}{c|}{$d_{2}=9 \mathrm{~nm}$} \\
\cline { 3 - 6 } & & Energy $E_{q}, \mathrm{eV}$ & $\begin{array}{c}\text { Activation energy } \\
E_{\text {act }}, \mathrm{eV}\end{array}$ & Energy $E_{q}, \mathrm{eV}$ & $\begin{array}{c}\text { Activation energy } \\
E_{\text {act, }}, \mathrm{eV}\end{array}$ \\
\hline CCCH & 0.458 & 0.838 & 0.384 & 0.766 & 0.350 \\
\hline CHHL & 3.758 & 0.916 & 3.444 & 0.803 & 3.018 \\
\hline CHHS & 4.089 & 0.477 & 1.951 & 0.405 & 1.655 \\
\hline CLLS & 0.749 & 0.555 & 0.416 & 0.442 & 0.331 \\
\hline
\end{tabular}

\section{RESULTS AND DISCUSSION}

For the asymmetric quantum-well heterostructure under consideration, the temperature dependence of the AR rate occurs to be activation-like. The effective energy of activation $E_{\text {act }}$ changes slightly with increasing the temperature and is affected by variations of $E_{q}$. Obviously, the energies $E_{q}$ decrease at the temperature growth in parallel with the decreasing of $E_{g}$. The main contribution to the total AR rate is introduced by the $\mathrm{CCCH}$ and CLLS processes. Such a behavior is associated with the lower specific $E_{\text {act }}$ that is caused with smaller values of $\Delta m$ as compared to other examined Auger processes. For the quantum wells with widths of 4-9 nm and for considered intervals of non-equilibrium carrier concentrations $n$ and temperatures $T$ the effective energy of activation $E_{\text {act }}$ lies in the range of 0.38 to $0.42 \mathrm{eV}$.

The results connected with the dependence $R(d)$ (Fig. 7) show that in the range of the quantum well width $d$ from 1 to $10 \mathrm{~nm}$ the AR rate increases since the levels structure changes. Firstly, the quantum-size level energy decreases. Secondly, it takes place the growth of the number of subbands in the quantum well and a new set of levels results in the AR rate raising in turn. Therewith, however, an activation-like temperature dependence of the AR rate remains.

Comparison of the contribution of spontaneous radiative recombination and non-radiative AR processes in the lasing threshold indicates that the quantum yield of spontaneous emission $\eta_{\mathrm{sp}}$ maintains sufficiently high at the threshold until the operation temperature $T_{\mathrm{op}}$ does not exceed $360 \mathrm{~K}$ and the cavity loss $k_{l}$ is lower than $80 \mathrm{~cm}^{-1}$. The suppressed role of the AR 208 Proc. of SPIE Vol. 5582 
in the threshold results from the conditions that the basic contribution to the spontaneous radiative recombination provides by the wider quantum well but the gain is determined in general by the narrower quantum well. In that way, the important practical result is the conclusion on the possibility of decreasing the AR rate influence on the temperature dependence $j_{\mathrm{th}}(T)$ in asymmetric multiple-quantum-well heterostructure lasers having a high-quality cavity and operating at room temperatures. Where $T_{\mathrm{op}}=360 \mathrm{~K}$ and $k_{1}=80 \mathrm{~cm}^{-1}$, the value of $\eta_{\mathrm{sp}}$ occurs to be of the order of 0.4 . In this case, taking into account $n=1$ and $E_{\text {act }} \approx 0.4 \mathrm{eV}$ one can evaluate according to relation (3) the value $T_{0} \approx 40 \mathrm{~K}$, i. e., the essential decreasing of the characteristic temperature parameter of the threshold is revealed.

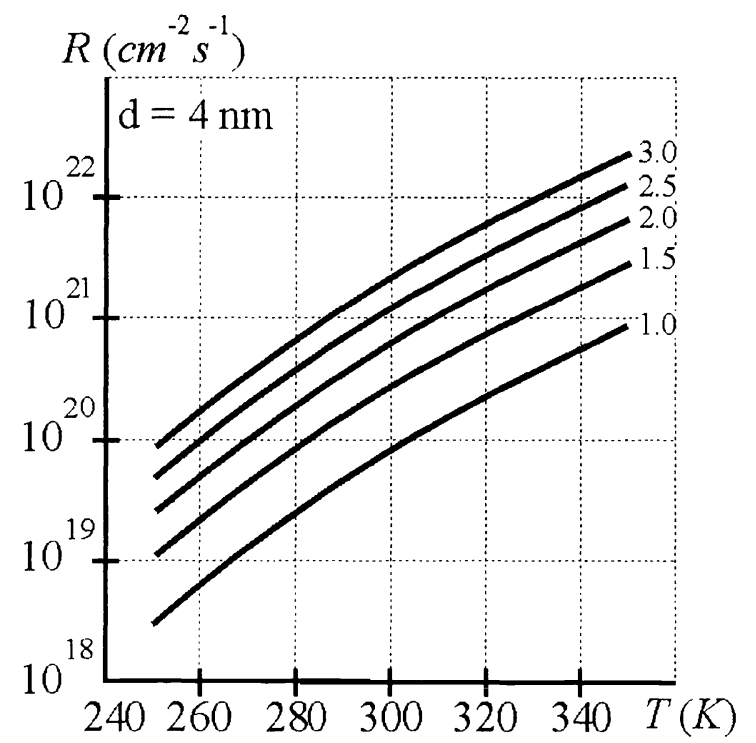

Figure 5: Dependence of the $\mathrm{AR}$ rate $R=C n^{3}$ on the temperature $T$ at different $n$ (numbers in $10^{13} \mathrm{~cm}^{-2}$ at the curves), $d=4 \mathrm{~nm}$.

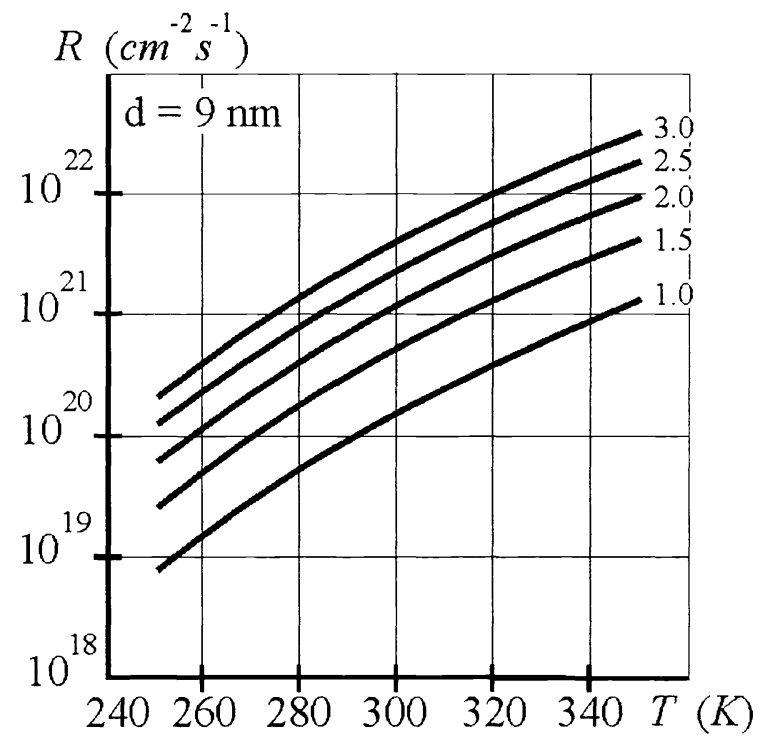

Figure 6: Dependence of the $\mathrm{AR}$ rate $R=C n^{3}$ on the temperature $T$ at different $n$ (numbers in $10^{13} \mathrm{~cm}^{-2}$ at the curves), $d=9 \mathrm{~nm}$.

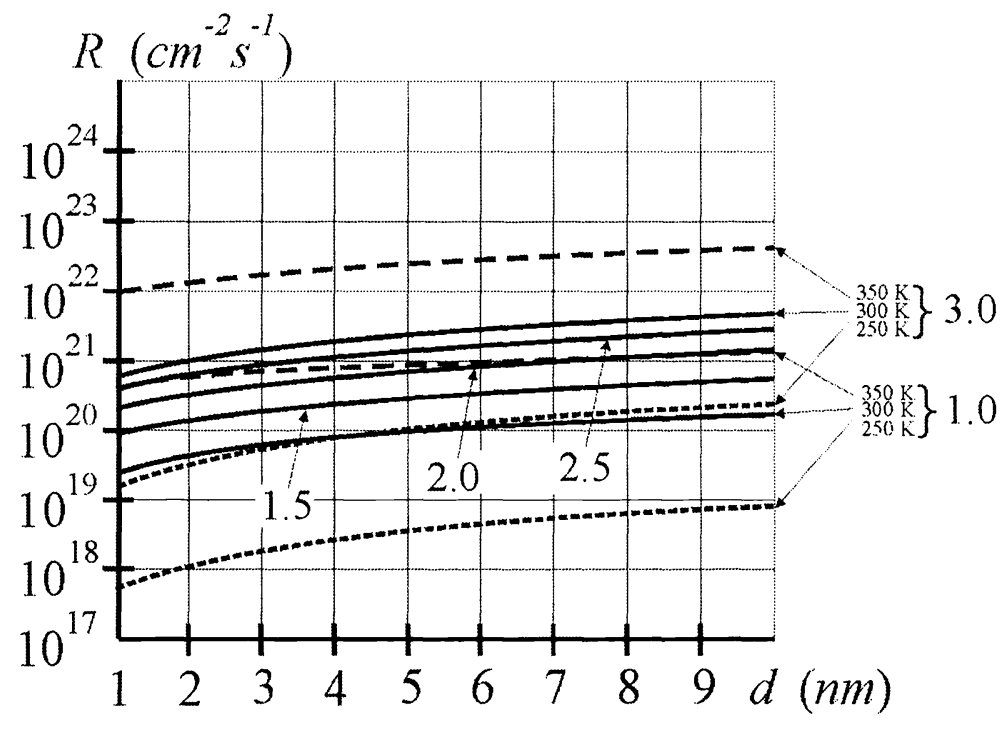

Figure 7: Dependence of the AR rate $R=C n^{3}$ on the quantum well width $d$ at different $n$ (numbers in $10^{13} \mathrm{~cm}^{-2}$ at the curves) and temperature $T=300 \mathrm{~K}$, other temperature values are shown outside. 


\section{CONCLUSION}

Asymmetric multiple-quantum-well heterostructure lasers possess lower temperature sensitivity of the threshold and reduced role of the AR. The most important contribution of the AR rate to the threshold of the GaInAs-GaInAsP-InP heterolasers emitted at the wavelength near $1.55 \mu \mathrm{m}$ is attributed with the CCCH and CLLS processes. The AR rate increases practically as an activation-like function in the temperature interval 250 to $350 \mathrm{~K}$ and follows an exponential dependence on the quantum well width $d$ varied from 4 to $9 \mathrm{~nm}$. As shown for the described asymmetric quantum-well heterostructure lasers the influence of AR processes on the temperature behavior of the lasing threshold is not essential until the temperature of the active region is lower than $360 \mathrm{~K}$ and the cavity losses does not exceed $80 \mathrm{~cm}^{-1}$. The presented analytical approach allows to evaluate the characteristic temperature of the lasing threshold and its relation with the effective activation energy of the AR processes.

\section{REFERENCES}

1. G. P. Agrawal, N. K. Dutta, Long-Wavelength Semiconductor Lasers. John Wiley \& Sons, N.-Y., 1986.

2. V. K. Kononenko, A. A. Afonenko, I. S. Manak, S. V. Nalivko, "Asymmetric multiple quantum well heterostructure laser systems: conception, performance, and characteristics," Opto-Electron. Rev. 2000. Vol. 3, No. 3. P. 241-250.

3. V. K. Kononenko, I. S. Manak, S. V. Nalivko, "Design and characteristics of widely tunable quantum-well laser diodes," Spectrochimica Acta, Part A: Mol. \& Biomol. Spectroscopy. 1999. Vol. 55, No. 10. P. 2091-2096.

4. V. K. Kononenko, Optical Properties of Heterostructures with Quantum-Size Layers. Preprint No. 492. IP AS BSSR, Minsk, 1987.

5. A. A. Afonenko, I. S. Manak, V. A. Shevtsov, V. K. Kononenko, "Radiative recombination rate in quantum-well structures in the model without k-selection," Semiconductors. 1997. Vol. 31, No. 9. P. 929-932.

6. A. D. Andreev, G. G. Zegrya, "Auger recombination in strained quantum wells," Semiconductors. 1997. Vol. 31, No. 3. P. 358-364.

7. Z.-M. Li, T. Bradford, "A comparative study of temperature sensitivity of InGaAsP and AlGaAs MQW lasers using numerical simulations," IEEE J. Quantum Electron. 1995. Vol. 31, No. 10. P. 1841-1847.

8. S. L. Chuang, "Efficient band-structure calculations of strained quantum wells," Phys. Rev. B. 1991. Vol. 43, No. 12. P. 9649-9661.

9. S. V. Nalivko, I. S. Manak, A. L. Chizh, "Influence of the band mixing effect on band structure and gain spectra of multiple-quantum-well heterostructures," Lithuanian J. Phys. 1999. Vol. 39, Nos. 4-5. P. 365-373.

10. V. K. Kononenko, S. V. Nalivko, "Spectral characteristics of asymmetric quantum-well heterostructure laser sources," Proc. SPIE. 1996. Vol. 2693. P. 760-767. 